\title{
Impulsive Evolution Equations with Causal Operators
}

\author{
Tahira Jabeen ${ }^{1}$, Ravi P. Agarwal ${ }^{2, *}$, Vasile Lupulescu ${ }^{3} \mathbb{D}$ and Donal $\mathrm{O}^{\prime}$ Regan $^{4}$ \\ 1 Department of Mathematics, International Islamic University, Sector H-10 Islamabad, Pakistan; \\ tahirajabeen14@gmail.com \\ 2 Department of Mathematics, Texas A\&M University-Kingville, Kingsville, TX 78363, USA \\ 3 Department of Mathematics, Constantin Brancusi University, Republicii 1, 210152 Targu-Jiu, Romania; \\ lupulescu_v@yahoo.com \\ 4 School of Mathematics, Statistics and Applied Mathematics, National University of Ireland, \\ H91 TK33 Galway, Ireland; donal.oregan@nuigalway.ie \\ * Correspondence: ravi.agarwal@tamuk.edu; Tel.: +1-361-593-3191
}

Received: 6 November 2019; Accepted: 20 December 2019; Published: 25 December 2019

\begin{abstract}
In this paper, we establish sufficient conditions for the existence of mild solutions for certain impulsive evolution differential equations with causal operators in separable Banach spaces. We rely on the existence of mild solutions for the strongly continuous semigroups theory, the measure of noncompactness and the Schauder fixed point theorem. We consider the impulsive integro-differential evolutions equation and impulsive reaction diffusion equations (which could include symmetric kernels) as applications to illustrate our main results.
\end{abstract}

Keywords: impulsive evolution equation; measure of noncompactness; existence result

\section{Introduction}

Let $\mathbb{R}$ be the set of real numbers and let $\mathbb{R}_{+}$be the set of non-negative real numbers. Let $E$ be a real Banach space endowed with the norm $\|\cdot\|$. We denote by $C([0, T], E)$ the Banach space of continuous functions from $[0, T]$ into $E$ endowed with the norm $\|u(\cdot)\|=\sup _{0 \leq t \leq a}\|u(t)\|$. The space of all strongly measurable functions $u(\cdot):[0, T] \rightarrow E$ such that

$$
\|u(\cdot)\|_{p}:=\left(\int_{0}^{T}\|u(t)\|^{p}\right)^{1 / p}<\infty
$$

for $1 \leq p<\infty$ and $\|u(\cdot)\|_{\infty}:=$ ess sup $\|u(t)\|<\infty$, will be denoted by $L^{p}([0, T], E)$. This is $t \in[0, T]$

a Banach space with respect to the norm $\|u(\cdot)\|_{p}$. Let $P C([0, T], E)$ be the set of all functions $u(\cdot):[0, T] \rightarrow E$ such that $u(\cdot)$ is continuous at $t \neq t_{k}$, left continuous at $t=t_{k}$ and the right limit $u\left(t_{k}^{+}\right)$exists for $k=1,2, \ldots, N$. Then $P C([0, T], E)$ is a Banach space with respect to the norm $\|u(\cdot)\|_{P C}:=\sup \{\|u(t)\| ; t \in[0, T]\}$. Moreover, we have that $P C([0, T], E) \subset L^{p}([0, T], E)$ and

$$
\|u(\cdot)\|_{1} \leq T^{1-1 / p}\|u(\cdot)\|_{p} \leq T^{2-1 / p}\|u(\cdot)\|_{P C} .
$$

Let us denote by $\mathcal{F}_{1}([0, T], X)$ the space of all the functions from $[0, T]$ into $X$, and by $\mathcal{F}_{2}([0, T], Y)$ the space of all the functions from $[0, T]$ into $Y$. Then an operator $\mathfrak{C}: \mathcal{F}_{1}([0, T], X) \rightarrow \mathcal{F}_{2}([0, T], Y)$ is called a causal operator if for each $\tau \in(0, T)$ and for all $u(\cdot), v(\cdot) \in \mathcal{F}_{1}([0, T], X)$ such that $u(t)=v(t)$ for $t \in[0, \tau]$, we have that $(\mathfrak{C} u)(t)=(\mathfrak{C} v)(t)$ for $t \in[0, \tau]$, and $(\mathfrak{C} 0)(t)=0$ for all $t \in[0, T]$. 
The aim of this paper is to establish existence results for mild solutions of the following impulsive evolution equation with the causal operator

$$
\left\{\begin{array}{l}
u^{\prime}(t)=A u(t)+(\mathfrak{C} u)(t) \text { for } t \in[0, T] \backslash\left\{t_{1}, \ldots, t_{N}\right\} \\
u\left(t_{k}^{+}\right)=u\left(t_{k}^{-}\right)+\left(\mathfrak{I}_{k} u\right)\left(t_{k}^{-}\right) \\
u(0)=u_{0}
\end{array}\right.
$$

where $A: D(A) \subset E \rightarrow E$ is the infinitesimal generator of a $C_{0}$-semigroup $\{\mathcal{T}(t) ; t \geq 0\}$ and $\mathfrak{C}: P C([0, T], E) \rightarrow L^{p}([0, T], E)$ is a continuous causal operator; here $1 \leq p \leq \infty, N \in \mathbb{N}, 0=t_{0}<$ $t_{1}<t_{2}<\ldots<t_{N}<t_{N+1}=T$ and $\Im_{k}: P C([0, T], E) \rightarrow P C([0, T], E)$ is a continuous causal operator for each $k=1,2, \ldots, N$.

The theory of differential equations involving causal operators allows a unified treatment for general classes of differential equations, such as: Ordinary differential equations, differential equations with delay, integro-differential equations, Volterra integral equations and so on. The term causal operator (or Volterra abstract operator) was introduced by Tonelli [1], and the theory of these classes of operators was developed by Tychonoff [2]. The class of causal operators is quite large and it includes a number of operators that are used in mathematical modeling of some phenomena in engineering and physics. An important class of causal operators is the class of superposition operators or Nemytskij operators (see [3]) $\mathfrak{C}: L^{p}([0, T], E) \rightarrow L^{p}([0, T], E)$ defined by $(\mathfrak{C} u)(t):=F(t, u(t))$, where $F:[0, T] \times E \rightarrow E$ is a Caratheodory function. If $\sigma>0$, then $\mathfrak{C}: C([-\sigma, T], E) \rightarrow L^{p}([0, T], E)$ defined by $(\mathfrak{C} u)(t):=F(t, u(t), u(t-\sigma))$ is another example of a causal operator. A more general example of causal operators is the operator $\mathfrak{C}: C([-\sigma, T], E) \rightarrow L^{p}([0, T], E)$ defined by

$$
(\mathfrak{C} u)(t):=F\left(t, u(t), u(t-\sigma), \int_{t-\sigma}^{t} K(t, s, u(s)) d s\right) .
$$

Several examples of causal operators and their applications can be found in the monograph [4]. Although it does not specifically study the theory of causal operators, several monographs, such as [5-9], address some aspects of differential equations involving causal operators. Detailed studies on differential equations with causal operators in finite dimensional spaces can be found in the monographs [4,10-12]. Applications of differential equations with causal operators in optimal control, adaptive control or hysteresis phenomena can be found in the papers [13-20]. Theoretical aspects regarding existence, stability or periodicity of solutions of differential equations with causal operators in finite or infinite dimensional spaces were presented in a series of works, such as: [21-39].

The study of evolution equations with causal operators was first presented in [40], where an existence result was obtained and some applications were given, but impulsive evolution differential equations with causal operators has not yet been studied. In this paper we study the class of impulsive evolution equations involving causal operators. In Section 2 we recall some results on $C_{0}$-semigroups of linear operators and some properties of the Hausdorff measure of noncompactness. In Section 3 we obtain the existence of mild solutions for a class of impulsive evolution equations with causal operators. Also, we show that a mild solution can be obtained as the limit of a sequence of successive approximations. In the last section we give some applications.

\section{Preliminaries}

We denote the space of all bounded linear operators acting on a Banach space $E$ by $\mathcal{L}(E)$. We recall that a family $\{\mathcal{T}(t) ; t \geq 0\} \subset \mathcal{L}(E)$ is called a $C_{0}$-semigroup if the following three properties are satisfied:

(a) $\mathcal{T}(0)=I$, the identity operator on $E$;

(b) $\mathcal{T}(t) \mathcal{T}(s)=\mathcal{T}(t+s)$ for all $t, s \geq 0$;

(c) $\lim _{t \rightarrow 0^{+}} \mathcal{T}(t) u=u$ for all $u \in E$. 
The infinitesimal generator of the $C_{0}$-semigroup $\{\mathcal{T}(t) ; t \geq 0\}$ is the operator $A: D(A) \subset E \rightarrow E$, defined by

$$
D(A)=\left\{u \in E ; \lim _{h \rightarrow 0^{+}} \frac{\mathcal{T}(h) u-u}{h} \text { exists }\right\}
$$

and

$$
A u=\lim _{h \rightarrow 0^{+}} \frac{\mathcal{T}(h) u-u}{h}, u \in D(A) .
$$

The generator is always a closed, densely defined operator. For further details on the theory of the $C_{0}$-semigroups see $[41,42]$.

We denote by $\chi(B)$ the Hausdorff measure of non-compactness of a nonempty bounded set $B \subset E$, and it is defined by [43]:

$$
\chi(B)=\inf \{\varepsilon>0 ; B \text { admits a finite cover by balls of radius } \leq \varepsilon\} .
$$

We recall some properties of $\chi$ (see [43]). If $A, B$ are bounded subsets of $E$, then

$(\chi 1) \chi(B)=0$ if and only if $\bar{B}$ is compact;

$(\chi 2) \chi(B)=\chi(\bar{B})=\chi(\overline{\operatorname{conv}}(B))$;

$(\chi 3) \chi(\lambda B)=|\lambda| \chi(B)$ for every $\lambda \in \mathbb{R}$;

$(\chi 4) \chi(B) \leq \chi(C)$ if $B \subset C$;

$(\chi 5) \chi(\{x\} \cup B)=\chi(B)$ for every $x \in E$;

$(\chi 6) \chi(B+C)=\chi(B)+\chi(C)$.

$(\chi 7)$ Generalized Cantor's intersection property : If $\left\{B_{n}\right\}_{n \geq 1}$ is a decreasing sequence of bounded closed nonempty subsets of $E$ and $\lim _{n \rightarrow \infty} \chi\left(B_{n}\right)=0$, then $\bigcap_{n=1}^{\infty} B_{n}$ is a nonempty and compact subset of $E$ (see [44]).

Remark 1. If $\operatorname{diam}(B)=\sup \{\|x-y\| ; x, y \in B\}$ is the diameter of the bounded set $A$, then we have that $\chi(B) \leq \operatorname{diam}(B)$ and $\chi(B) \leq 2 d$ if $\sup _{x \in B}\|x\| \leq d$.

In the following, we denote by $\chi_{c}$ the Hausdorff measure of non-compactness in the space $C([0, T], E)$. Then it is well known that for every bounded set $B \subset C([0, T], E)$ we have

$$
\chi(B(t)) \leq \chi_{c}(B)
$$

for every $t \in[0, T]$, where $B(t):=\{u(t): u \in B\}$. Moreover, for every bounded and equicontinuous set $B \subset C([0, T], E)$ we have (see [43])

$$
\chi_{c}(B)=\sup _{0 \leq t \leq T} \chi(B(t))
$$

For each $k=0,1,2, \ldots, N$ and $u(\cdot) \in P C([0, T], E)$ we set $J_{k}=\left(t_{k}, t_{k+1}\right], \bar{J}_{k}=\left[t_{k}, t_{k+1}\right]$ and introduce the function $\widetilde{u}_{k}(\cdot) \in C\left(\bar{J}_{k}, E\right)$ defined by

$$
\widetilde{u}_{k}(t)=\left\{\begin{array}{l}
u(t), \text { for } t \in J_{k} \\
u\left(t_{k}^{+}\right), \text {for } t=t_{k}
\end{array}\right.
$$


Also, for $B \subset P C([0, T], E)$ and $k=0,1,2, \ldots, N$, let us set $\widetilde{B}^{k}:=\left\{\widetilde{u}_{k}(\cdot) \in C\left(\bar{J}_{k}, E\right) ; u(\cdot) \in B\right\}$. If we denote by $\chi_{k}$ the Hausdorff measure of noncompactness on $C\left(\bar{J}_{k}, E\right)$, then

$$
\chi_{P C}(B):=\max _{0 \leq k \leq N} \chi_{k}\left(\widetilde{B}^{k}\right), B \subset P C([0, T], E)
$$

defines the Hausdorff measure of noncompactness on $P C([0, T], E)$. Moreover, it is easy to see that

$$
\chi_{P C}(B)=\sup _{t \in[0, T]} \chi(B(t))
$$

for every equicontinuous subset $B \subset P C([0, T], E)$.

Lemma 1 ([45], Lemma 2.1). A set $B \subset P C([0, T], E)$ is relatively compact in $P C([0, T], E)$ if and only if $\widetilde{B}^{k}$ is relatively compact in $C\left(\bar{J}_{k}, E\right)$ for every $k=0,1,2, \ldots, N$.

Lemma 2 ([46], p. 125). If $B \subset E$ is a nonempty bounded set, then for every $\varepsilon>0$ there exists a sequence $\left\{x_{n}\right\}_{n \geq 1}$ in E such that

$$
\chi(B) \leq 2 \chi\left(\left\{x_{n} ; n \geq 1\right\}\right)+\varepsilon .
$$

Lemma 3 ([47], Lemma 2.2). Let $\left\{u_{n}(\cdot) ; n \geq 1\right\}$ be a subset in $L^{1}([0, T], E)$ for which there exists $m(\cdot) \in$ $L^{1}\left([0, T], \mathbb{R}_{+}\right)$such that $\left\|u_{n}(t)\right\| \leq m(t)$ for each $n \geq 1$ and for a.e. $t \in[0, T]$. Then the function $t \mapsto$ $\chi(t):=\chi\left(\left\{u_{n}(t) ; n \geq 1\right\}\right)$ is integrable on $[0, T]$ and, for each $t \in[0, T]$, we have

$$
\chi\left(\left\{\int_{0}^{t} u_{n}(s) d s ; n \geq 1\right\}\right) \leq \int_{0}^{t} \chi(s) d s .
$$

\section{Existence Result}

Consider the following impulsive differential equation

$$
\left\{\begin{array}{l}
u^{\prime}(t)=A u(t)+(\mathfrak{C} u)(t) \text { for } t \in[0, T] \backslash\left\{t_{1}, \ldots, t_{N}\right\} \\
u\left(t_{k}^{+}\right)=u\left(t_{k}^{-}\right)+\left(\mathfrak{I}_{k} u\right)\left(t_{k}^{-}\right) \\
u(0)=u_{0}
\end{array}\right.
$$

where $A: D(A) \subset E \rightarrow E$ is the infinitesimal generator of a $C_{0}$-semigroup $\{\mathcal{T}(t) ; t \geq 0\}$ and $\mathfrak{C}: P C([0, T], E) \rightarrow L^{p}([0, T], E)$ is a continuous causal operator; here $1 \leq p \leq \infty, N \in \mathbb{N}, 0=t_{0}<$ $t_{1}<t_{2}<\ldots<t_{N}<t_{N+1}=T$ and $\mathfrak{I}_{k}: P C([0, T], E) \rightarrow P C([0, T], E)$ is a continuous causal operator for each $k=1,2, \ldots, N$.

A function $u(\cdot) \in P C([0, T], E)$ is called a mild solution of (4) if it satisfies

$$
u(t)=\mathcal{T}(t) u(0)+\int_{0}^{t} \mathcal{T}(t-s)(\mathfrak{C} u)(s) d s+\sum_{0<t_{k}<t} \mathcal{T}\left(t-t_{k}\right) \mathfrak{I}_{k}\left(u\left(t_{k}\right), t \in[0, T] .\right.
$$

Let us introduce the following conditions.

(H1) For each $k=1,2, \ldots, N, \Im_{k}: P C([0, T], E) \rightarrow P C([0, T], E)$ is continuous and a compact operator and there exists $c_{k}>0$, with $M \sum_{0<t_{k}<T} c_{k}<1$, such that for each $u(\cdot) \in P C([0, T], E)$ we have

$$
\left\|\left(\Im_{k} u\right)(t)\right\| \leq c_{k}\|u(t)\| \text { for every } t \in[0, T],
$$

where $M:=\sup _{0 \leq t \leq T}\|\mathcal{T}(t)\|$. 
(H2) (a) There exists a function $\xi(\cdot, \cdot):[0, T] \times \mathbb{R}_{+} \rightarrow \mathbb{R}_{+}$such that $\xi(\cdot, \eta) \in L^{1}\left([0, T], \mathbb{R}_{+}\right)$for every $\eta \in \mathbb{R}_{+}, \xi(t, \cdot)$ is continuous and increasing on $\mathbb{R}_{+}$for a.e. $t \in[0, T]$ such that

$$
\limsup _{\eta \rightarrow \infty} \frac{M}{\eta}\left(\left\|u_{0}\right\|+\int_{0}^{T} \xi(s, \eta) d s\right)<1-M \sum_{0<t_{k}<T} c_{k}
$$

and

$$
\|(\mathfrak{C} u)(t)\| \leq \xi(t,\|u(t)\|), \text { for a.e. } t \in[0, T],
$$

for each $u(\cdot) \in P C([0, T], E)$.

(b) For each bounded subsets $B \subset P C([0, T], E)$ there exists $\gamma_{B}(\cdot) \in L^{1}\left([0, T], \mathbb{R}_{+}\right)$such that

$$
\int_{0}^{T} \gamma_{B}(t) d t<\frac{1}{2 M T}
$$

and

$$
\chi((\mathfrak{C} B)(t)) \leq \int_{0}^{t} \gamma_{B}(s) \chi(B(s)) d s \text { for } t \in[0, T],
$$

where $(\mathfrak{C} B)(t):=\{(\mathfrak{C} u)(t): u(\cdot) \in B\}$.

Theorem 1. Let $\mathfrak{C}: P C([0, T], E) \rightarrow L^{p}([0, T], E)$ be a continuous causal operator such that conditions (H1) and (H2) hold. If $A: D(A) \subset E \rightarrow E$ is the generator of a $C_{0}$-semigroup $\{\mathcal{T}(t) ; t \geq 0\}$, then the evolution Equation (4) has at least one mild solution on $[0, T]$.

Proof. First, we remark that there exists an $r>0$ such that

$$
M\left\|u_{0}\right\|+M \int_{0}^{T} \xi(s, r) d s+M r \sum_{0<t_{k}<T} c_{k}<r .
$$

Indeed, from (5) it follows that there exists $\eta_{0}>0$ such that

$$
\frac{M}{\eta}\left(\left\|u_{0}\right\|+\int_{0}^{T} \xi(s, \eta) d s\right)<1-M \sum_{0<t_{k}<T} c_{k}
$$

for every $\eta>\eta_{0}$, so that

$$
M\left\|u_{0}\right\|+M \int_{0}^{T} \xi(s, \eta) d s+M \eta \sum_{0<t_{k}<T} c_{k}<\eta
$$

for every $\eta>\eta_{0}$. Consequently, we can choose a $r>\eta_{0}$ such that (9) holds. Now, let

$$
B_{0}=\left\{u(\cdot) \in P C([0, T], E) ;\|u(\cdot)\|_{P C} \leq r\right\},
$$

and define the operator $\mathfrak{F}: B_{0} \rightarrow P C([0, T]), E$ by

$$
(\mathfrak{F} u)(t):=\mathcal{T}(t) u_{0}+\int_{0}^{t} \mathcal{T}(t-s)(\mathfrak{C} u)(s) d s+\sum_{0<t_{k}<t} \mathcal{T}\left(t-t_{k}\right)\left(\mathfrak{I}_{k} u\right)\left(t_{k}\right),
$$


for $t \in[0, T]$. Since $\xi(t, \cdot)$ is increasing on $\mathbb{R}_{+}$for a.e. $t \in[0, T]$ for every $u(\cdot) \in B_{0}$, using (5) we have

$$
\begin{aligned}
& \|(\mathfrak{F} u)(t)\| \leq\left\|\mathcal{T}(t) u_{0}\right\|+\int_{0}^{t}\|\mathcal{T}(t-s)(\mathfrak{C} u)(s)\| d s+\sum_{0<t_{k}<t}\left\|\mathcal{T}\left(t-t_{k}\right)\left(\mathfrak{I}_{k} u\right)\left(t_{k}\right)\right\| \\
& \leq M\left\|u_{0}\right\|+M \int_{0}^{t}\|(\mathfrak{C} u)(s)\| d s+M \sum_{0<t_{k}<t} c_{k}\left\|u\left(t_{k}\right)\right\| \\
& \leq M\left\|u_{0}\right\|+M \int_{0}^{t} \xi(s,\|u(s)\|) d s+M \sum_{0<t_{k}<t} c_{k}\left\|u\left(t_{k}\right)\right\| \\
& \leq M\left\|u_{0}\right\|+M \int_{0}^{T} \xi(s,\|u(s)\|) d s+M \sum_{0<t_{k}<T} c_{k}\left\|u\left(t_{k}\right)\right\| \\
& \leq M\left\|u_{0}\right\|+M \int_{0}^{T} \xi(s, r) d s+M r \sum_{0<t_{k}<T} c_{k}<r
\end{aligned}
$$

so that $\mathfrak{F}\left(B_{0}\right) \subset B_{0}$. We notice that $\|(\mathfrak{C} u)(t)\| \leq \psi(t)$ for a.e. on $[0, T]$, for every $u(\cdot) \in B_{0}$, where $\psi(\cdot):=\mathfrak{\xi}(\cdot, r) \in L^{1}\left([0, T], \mathbb{R}_{+}\right)$. Let $B_{1}:=\mathfrak{F} B_{0}$. Next, we will show that $\widetilde{B}_{1}^{k}$ is equicontinuous on $\bar{J}_{k}$ for every $k=1,2, \ldots, N$. For this, we shall write the operator $\mathfrak{F}$ as $(\mathfrak{F} u)(t)=\left(\mathfrak{F}_{1} u\right)(t)+$ $\left(\mathfrak{F}_{2} u\right)(t)$, where

$$
\begin{gathered}
\left(\mathfrak{F}_{1} u\right)(t)=\mathcal{T}(t) u_{0}+\int_{0}^{t} \mathcal{T}(t-\tau)(\mathfrak{C} u)(\tau) d \tau, \\
\left(\mathfrak{F}_{2} u\right)(t)=\sum_{0<t_{k}<t} \mathcal{T}\left(t-t_{k}\right)\left(\mathfrak{I}_{k} u\right)\left(t_{k}\right)
\end{gathered}
$$

for $t \in[0, T]$.

First, we show that $G_{1}:=\mathfrak{F}_{1} B_{0}$ is equicontinuous on $[0, T]$. Let $\varepsilon>0$. Since $t \mapsto \mathcal{T}(t) u_{0}$ continuous on $[0, T]$ (see [42], Corollary 2.3), then there exists $\delta_{1}=\delta_{1}(\varepsilon / 5)>0$ such that

$$
\left\|\mathcal{T}(t+h) u_{0}-\mathcal{T}(t) u_{0}\right\| \leq \frac{\varepsilon}{5}
$$

for every $t \in[0, T]$ and $h \in \mathbb{R}$ with $|h|<\delta_{1}$ and $t+h \in[0, T]$. On $[0, T]$, the function $t \mapsto \int_{0}^{t} \psi(s) d s$ is uniformly continuous and thus there exists $\delta_{2}=\delta_{2}(\varepsilon / 5 M)>0$ such that

$$
\left|\int_{t}^{t+h} \psi(\tau) d \tau\right|<\frac{\varepsilon}{5 M}
$$

for every $t \in[0, T]$ and $h \in \mathbb{R}$ with $|h|<\delta_{2}$ and $t+h \in[0, T]$. Then for $t=0$ we have

$$
\begin{aligned}
& \left\|\left(\mathfrak{F}_{1} u\right)(h)-\left(\mathfrak{F}_{1} u\right)(0)\right\|=\| \mathcal{T}(h) u_{0}+\int_{0}^{h}\left[\mathcal{T}(h-\tau)(\mathfrak{C} u)(\tau) d \tau-u_{0} \|\right. \\
& \leq\left\|\mathcal{T}(h) u_{0}-u_{0}\right\|+\int_{0}^{h}\|\mathcal{T}(h-\tau)(\mathfrak{C} u)(\tau)\| d \tau \leq\left\|\mathcal{T}(h) u_{0}-u_{0}\right\| \\
& +M \int_{0}^{h} \leftarrow(\tau) d \tau<\frac{2 \varepsilon}{5}<\varepsilon
\end{aligned}
$$

for each $u \in B_{0}$ and $h \in(0, T]$ with $h<\min \left\{\delta_{1}, \delta_{2}\right\}$. It follows that $G_{1}$ is equicontinuous at $t=0$. Next, take $t \in(0, T]$ and let us choose $0<\eta<\delta_{2} / 2$ such that $t-\eta \in[0, T]$. For each $u \in B_{0}$ and $h \in \mathbb{R}$ such that $t+h \in[0, T]$ we have 


$$
\begin{aligned}
& \left\|\left(\mathfrak{F}_{1} u\right)(t+h)-\left(\mathfrak{F}_{1} u\right)(t)\right\| \leq\left\|\left(\mathfrak{F}_{1} u\right)(t)-\mathcal{T}(\eta)\left[\left(\mathfrak{F}_{1} u\right)(t-\eta)\right]\right\| \\
& +\left\|\mathcal{T}(\eta)\left[\left(\mathfrak{F}_{1} u\right)(t-\eta)\right]-\mathcal{T}(\eta+h)\left[\left(\mathfrak{F}_{1} u\right)(t-\eta)\right]\right\| \\
& +\left\|\mathcal{T}(\eta+h)\left[\left(\mathfrak{F}_{1} u\right)(t-\eta)\right]-\left(\mathfrak{F}_{1} u\right)(t+h)\right\|
\end{aligned}
$$

Since

$$
\begin{aligned}
& \left\|\left(\mathfrak{F}_{1} u\right)(t)-\mathcal{T}(\eta)\left[\left(\mathfrak{F}_{1} u\right)(t-\eta)\right]\right\|=\| \mathcal{T}(t) u_{0}+\int_{0}^{t} \mathcal{T}(t-\tau)(\mathfrak{C} u)(\tau) d \tau \\
& -\mathcal{T}(\eta)\left[\mathcal{T}(t-\eta) u_{0}+\int_{0}^{t-\eta}[\mathcal{T}(t-\eta-\tau)(\mathfrak{C} u)(\tau) d \tau] \|\right. \\
& =\left\|\int_{0}^{t} \mathcal{T}(t-\tau)(\mathfrak{C} u)(\tau) d \tau-\int_{0}^{t-\eta} \mathcal{T}(t-\tau)(\mathfrak{C} u)(\tau) d \tau\right\| \\
& =\left\|\int_{t-\eta}^{t} \mathcal{T}(t-\tau)(\mathfrak{C} u)(\tau) d \tau\right\| \leq M \int_{t-\eta}^{t} \psi(\tau) d \tau
\end{aligned}
$$

it follows that

$$
\left\|\left(\mathfrak{F}_{1} u\right)(t)-\mathcal{T}(\eta)\left[\left(\mathfrak{F}_{1} u\right)(t-\eta)\right]\right\| \leq M \int_{t-\eta}^{t} \psi(\tau) d \tau<\frac{\varepsilon}{5}
$$

for each $u \in B_{0}$. By similar reasoning, we obtain

$$
\left\|\mathcal{T}(\eta+h)\left[\left(\mathfrak{F}_{1} u\right)(t-\eta)\right]-\left(\mathfrak{F}_{1} u\right)(t+h)\right\| \leq M \int_{t-\eta}^{t+h} \psi(\tau) d \tau,
$$

and so, by (13), we conclude that

$$
\left\|\mathcal{T}(\eta+h)\left[\left(\mathfrak{F}_{1} u\right)(t-\eta)\right]-\left(\mathfrak{F}_{1} u\right)(t+h)\right\| \leq M \int_{t-\eta}^{t+h} \psi(\tau) d \tau<\frac{\varepsilon}{5}
$$

for each $u \in B_{0}$ and $h \in \mathbb{R}$ with $|h|<\eta$ and $t+h \in[0, T]$. Furthermore, we have

$$
\begin{aligned}
& \left\|\mathcal{T}(\eta)\left[\left(\mathfrak{F}_{1} u\right)(t-\eta)\right]-\mathcal{T}(\eta+h)\left[\left(\mathfrak{F}_{1} u\right)(t-\eta)\right]\right\| \leq\left\|\mathcal{T}(t+h) u_{0}-\mathcal{T}(t) u_{0}\right\| \\
& +\int_{0}^{t-\eta}\|\mathcal{T}(t+h-\tau)-\mathcal{T}(t-\tau)\| \psi(\tau) d \tau \leq\left\|\mathcal{T}(t+h) u_{0}-\mathcal{T}(t) u_{0}\right\| \\
& +2 M \int_{0}^{t-\eta} \psi(\tau) d \tau \leq \frac{3 \varepsilon}{5}
\end{aligned}
$$

that is,

$$
\left\|\mathcal{T}(\eta)\left[\left(\mathfrak{F}_{1} u\right)(t-\eta)\right]-\mathcal{T}(\eta+h)\left[\left(\mathfrak{F}_{1} u\right)(t-\eta)\right]\right\| \leq \frac{2 \varepsilon}{5}
$$

for each $u \in B_{0}$ and $h \in \mathbb{R}$ with $|h|<\min \left\{\eta, \delta_{1}, \delta_{2}\right\}$ and $t+h \in[0, T]$. Now, using (15), (16) and (18), from (14) it follows that

$$
\left\|\left(\mathfrak{F}_{1} u\right)(t+h)-\left(\mathfrak{F}_{1} u\right)(t)\right\|<\varepsilon
$$

for each $u \in B_{0}$ and $h \in \mathbb{R}$ with $|h|<\min \left\{\eta, \delta_{1}, \delta_{2}\right\}$ and $t+h \in[0, T]$. Thus $G_{1}$ is equicontinuous on $[0, T]$. From this it follows that $\widetilde{G}_{1}^{k}$ is equicontinuous on $\bar{J}_{k}$ for every $k=1,2, \ldots, N$. Next, we show that, for a given $v \in\{1,2, \ldots, N\}$, the set $\widetilde{G}_{2}^{v}$ is equicontinuous on $\bar{J}_{v}$, where $G_{2}:=\mathfrak{F}_{2} B_{0}$. Since $\mathfrak{I}_{k}$ is a compact operator, $\mathfrak{I}_{k} B_{0}$ is a relatively compact set in $\operatorname{PC}([0, T], E)$ and so, by Lemma $1 \mathfrak{I}_{k} \widetilde{B}_{0}$ is a relatively compact set in $C\left(\bar{J}_{k}, E\right)$ for each $k=1,2, \ldots, N$. Using the Ascoli-Arzela theorem, from the compactness of $\mathfrak{I}_{k} \widetilde{B}_{0}$ in $C\left(\bar{J}_{k}, E\right)$, it follows that $\left(\mathfrak{I}_{k} \widetilde{B}_{0}\right)(t)$ is relatively compact in $E$ for every $t \in \bar{J}_{k}$ and $k=1,2, \ldots, N$. In particular, $\left(\mathfrak{I}_{k} \widetilde{B}_{0}\right)\left(t_{k}\right)$ is relatively compact for every $k=1,2, \ldots, N$, and thus $K:=\bigcup_{k=1}^{N}\left(\mathfrak{I}_{k} \widetilde{B}_{0}\right)\left(t_{k}\right)$ is relatively compact in $E$. Since $(t, x) \mapsto T(t) x$ is jointly continuous from $[0, \infty) \times K$ to $E$, it follows that there exists a $\delta>0$ such that 


$$
\left\|T\left(t-t_{k}\right) x-T\left(s-t_{k}\right) x\right\|<\varepsilon / N, x \in K
$$

for every $t_{k}, k=1,2, \ldots, N, t, s \in \bar{J}_{k}$ with $|t-s|<\delta$. Next, for every $u(\cdot) \in B_{0}, t, s \in \bar{J}_{v}$ with $|t-s|<\delta$, we have

$$
\begin{aligned}
& \left\|\left(\mathfrak{F}_{2} \widetilde{u}_{v}\right)(t)-\left(\mathfrak{F}_{2} \widetilde{u}_{v}\right)(s)\right\|=\left\|\left(\mathfrak{F}_{2} u\right)(t)-\left(\mathfrak{F}_{2} u\right)(s)\right\| \\
& =\left\|\sum_{0<t_{k}<t} \mathcal{T}\left(t-t_{k}\right)\left(\mathfrak{I}_{k} u\right)\left(t_{k}\right)-\sum_{0<t_{k}<t} \mathcal{T}\left(s-t_{k}\right)\left(\mathfrak{I}_{k} u\right)\left(t_{k}\right)\right\| \\
& \leq \sum_{k=1}^{v}\left\|T\left(t-t_{k}\right)\left(\mathfrak{I}_{k} u\right)\left(t_{k}\right)-\mathcal{T}\left(s-t_{k}\right)\left(\mathfrak{I}_{k} u\right)\left(t_{k}\right)\right\|<\varepsilon,
\end{aligned}
$$

so that $\widetilde{G}_{2}^{v}$ is equicontinuous on $\bar{J}_{v}$. Since $\widetilde{B}_{1}^{k}=\widetilde{G}_{1}^{k}+\widetilde{G}_{2}^{k}, k=1,2, \ldots, N$, it follows that $\widetilde{B}_{1}^{k}$ is equicontinuous on $\bar{J}_{k}$ for every $k=1,2, \ldots, N$. Next, for each $n \geq 1$, we define $B_{n}=\overline{\operatorname{conv}}\left(\mathfrak{F} B_{n-1}\right)$. Then, for every $n \geq 1, B_{n} \subset P C([0, T], E)$ is a bounded, closed and convex set. Now, from $\mathfrak{F} B_{0} \subset B_{0}$, it follows that

$$
B_{1}=\overline{\operatorname{conv}}\left(\mathfrak{F} B_{0}\right) \subset \overline{\operatorname{conv}}\left(B_{0}\right)=B_{0} .
$$

If we suppose that $B_{v} \subset B_{v-1}$ for a given $v>1$, then

$$
B_{v+1}=\overline{\operatorname{conv}}\left(\mathfrak{F} B_{v}\right) \subset \overline{\operatorname{conv}}\left(\mathfrak{F} B_{v-1}\right)=B_{v}
$$

and thus, by induction it follows that $B_{n} \subset B_{n-1}$ for every $n \geq 1$. Moreover, it is easy to see that $\widetilde{B}_{n}^{k}$ is equicontinuous on $\bar{J}_{k}$ for each $k=1,2, \ldots, N$ and for every $n \geq 1$. Now, we will show that $\chi_{P C}\left(B_{n}\right) \rightarrow 0$ as $n \rightarrow \infty$. From Lemma 2, it follows that there exists a sequence $\left\{v_{m}(\cdot)\right\}_{m \geq 1}$ in $\mathfrak{F} B_{n-1}$ such that

$$
\chi_{P C}\left(B_{n}\right)=\chi_{P C}\left(\mathfrak{F} B_{n-1}\right) \leq 2 \chi_{P C}(V)+\varepsilon,
$$

where $V:=\left\{v_{m}(\cdot) ; m \geq 1\right\}$. From the above inequality it follows that

$$
\chi_{P C}\left(B_{n}\right) \leq 2 \max _{0 \leq k \leq N} \chi_{k}\left(\bar{V}_{n}^{k}\right)+\varepsilon .
$$

Since for each $k=1,2, \ldots, N$, the equicontinuity of $\bar{V}_{n}^{k}$ and Lemma 3 imply $\chi_{k}\left(\bar{V}_{n}^{k}\right)=\sup _{t \in J_{k}} \chi(V(t))$, we obtain

$$
\begin{aligned}
\chi_{P C}\left(B_{n}\right) & \leq 2 \max _{0 \leq k \leq N}\left[\sup _{t \in J_{k}} \chi(V(t))\right]+\varepsilon \leq 2 \sup _{t \in[0, T]} \chi(V(t))+\varepsilon \\
& =2 \sup _{t \in[0, T]} \chi\left(\left\{v_{m}(t) ; m \geq 1\right\}\right)+\varepsilon .
\end{aligned}
$$

Further, let $\left\{u_{m}(\cdot)\right\}_{m \geq 1}$ be a sequence in $B_{n-1}$ such that $v_{m}(\cdot)=\left(\mathfrak{F} u_{m}\right)(\cdot), m \geq 1$. If we put $V:=\left\{u_{m}(\cdot) ; m \geq 1\right\}$ and $V(t):=\left\{u_{m}(t) ; m \geq 1\right\}, t \in[0, T]$, then from the previous inequality it follows that 


$$
\begin{aligned}
\chi_{P C}\left(B_{n}\right) \leq & \varepsilon+2 \chi\left(\mathcal{T}(t-s) u_{0}+\int_{0}^{t} \mathcal{T}(t-s)(\mathfrak{C} V)(s) d s\right. \\
& \left.+\sum_{0<t_{k}<t-T / n} \mathcal{T}\left(t-t_{k}\right)\left(\mathfrak{I}_{k} V\right)\left(t_{k}\right)\right) \\
\leq & \varepsilon+2 \chi\left(\int_{0}^{t} \mathcal{T}(t-s)(\mathfrak{C} V)(s) d s\right) \\
& +2 \chi\left(\sum_{0<t_{k}<t} \mathcal{T}\left(t-t_{k}\right)\left(\mathfrak{I}_{k} V\right)\left(t_{k}\right)\right) .
\end{aligned}
$$

Let $t \in[0, T]$ be fixed. Since

$$
\left\|\mathcal{T}(t-s)\left(\mathfrak{C} u_{m}\right)(s)\right\| \leq M \psi(t) \text { for a.e. } s \in[0, t]
$$

for all $m \geq 1$, and

$$
\begin{aligned}
\chi\left(\left\{\left(\mathfrak{C} u_{m}\right)(s) ; m\right.\right. & \geq 1\}) \leq \int_{0}^{s} \gamma_{V}(\tau) \chi\left(\left\{u_{m}(\tau) ; m \geq 1\right\}\right) d \tau \\
& \leq \int_{0}^{s} \gamma_{V}(\tau) \chi\left(B_{n-1}(\tau)\right) d \tau \leq \chi_{P C}\left(B_{n-1}\right) \int_{0}^{s} \gamma_{V}(\tau) d \tau
\end{aligned}
$$

for a.e. $s \in[0, t]$, by Lemma 3 it follows that

$$
\begin{aligned}
\chi\left(\int_{0}^{t} \mathcal{T}(t-s)(\mathfrak{C} V)(s) d s\right) & \leq \int_{0}^{t} \chi(\mathcal{T}(t-s)(\mathfrak{C} V)(s)) d s \\
& \leq 2 M \int_{0}^{t} \chi((\mathfrak{C} V)(s)) d s \\
& \leq 2 M \int_{0}^{t} \int_{0}^{s} \gamma_{V}(\tau) \chi((V(\tau)) d \tau d s \\
& \leq 2 M \chi_{P C}\left(B_{n-1}\right) \int_{0}^{t} \int_{0}^{s} \gamma_{V}(\tau) d \tau d s \\
& =2 M \chi_{P C}\left(B_{n-1}\right) \int_{0}^{t} \int_{\tau}^{t} \gamma_{V}(\tau) d s d \tau \\
& =2 M \chi_{P C}\left(B_{n-1}\right) \int_{0}^{t}(t-\tau) \gamma_{V}(\tau) d \tau \\
& \leq 2 M T \chi_{P C}\left(B_{n-1}\right) \int_{0}^{T} \gamma_{V}(\tau) d \tau
\end{aligned}
$$

Also, by the continuity of the operators $T(t)$ and by the compactness of the operators $\mathfrak{I}_{k}$, it follows that the set $\left\{\mathcal{T}\left(t-t_{k}\right)\left(\mathfrak{I}_{k} V\right)\left(t_{k}\right)\right\}$ is relatively compact for every $t \in[0, T]$. Therefore, we have that

$$
\chi\left(\sum_{0<t_{k}<t} \mathcal{T}\left(t-t_{k}\right)\left(\Im_{k} V\right)\left(t_{k}\right)\right) \leq \sum_{0<t_{k}<t} \chi\left(\mathcal{T}\left(t-t_{k}\right)\left(\Im_{k} V\right)\left(t_{k}\right)\right)=0 .
$$

From (19), (21), and (22), we obtain

$$
\chi_{P C}\left(B_{n}\right) \leq \varepsilon+\rho \chi_{P C}\left(B_{n-1}\right)
$$

where

$$
\rho:=2 M T \int_{0}^{T} \gamma_{V}(s) d s<1
$$


Since $\varepsilon>0$ is arbitrary, it follows that

$$
\chi_{P C}\left(B_{n}\right) \leq \rho \chi_{P C}\left(B_{n-1}\right) .
$$

Also

$$
\chi_{P C}\left(B_{n}\right) \leq \rho^{n-1} \chi_{P C}\left(B_{1}\right) .
$$

Since the last inequality is true for every $n \geq 1$ and $0<\rho<1$, passing to the limit as $n \rightarrow \infty$, we obtain $\lim _{n \rightarrow \infty} \chi_{P C}\left(B_{n}\right)=0$. Now, using the generalized Cantor's intersection property, it follows that the set $B:=\bigcap_{n=1}^{\infty} B_{n}$ is a nonempty and compact subset of $P C([0, T], E)$. Since every set $B_{n}$ is a convex set, the set $B$ is also a convex set. Next, we verify that $\mathfrak{F} B \subset B$. Indeed, for every $n \geq 1$, we have that $\mathfrak{F} B \subset \mathfrak{F} B_{n} \subset \overline{\operatorname{conv}}\left(B_{n}\right)=B_{n+1}$, so that $\mathfrak{F} B \subset \bigcap_{n=2}^{\infty} B_{n}$. Also, since $B_{n} \subset B_{1}$ for every $n \geq 1$, it follows that $\mathfrak{F} B \subset \bigcap_{n=2}^{\infty} B_{n} \subset \bigcap_{n=1}^{\infty} B_{n} \subset B$. Now, we show that $\mathfrak{F}$ is a continuous operator from $B$ into itself. For this, let $u_{n}(\cdot) \rightarrow u(\cdot)$ in $B$. If $1 \leq p<\infty$ and $1 / p+1 / q=1$, then by Hölder's inequality we have

$$
\begin{aligned}
& \left\|\left(\mathfrak{F} u_{n}\right)(t)-(\mathfrak{F} u)(t)\right\| \\
\leq & \int_{0}^{t}\left[\left\|\mathcal{T}(t-s)\left[\left(\mathfrak{C} u_{n}\right)(s)-(\mathfrak{C} u)(s)\right]\right\| d s\right. \\
& \left.+\sum_{0<t_{k}<t}\left\|\mathcal{T}\left(t-t_{k}\right)\left(\mathfrak{I}_{k} u_{n}\right)\left(t_{k}\right)-\left(\mathfrak{I}_{k} u\right)\left(t_{k}\right)\right\|\right] \\
\leq & \int_{0}^{t}\|\mathcal{T}(t-s)\|_{\mathcal{L}(E)}\left\|\left(\mathfrak{C} u_{n}\right)(s)-(\mathfrak{C} u)(s)\right\| d s \\
& \left.+\sum_{0<t_{k}<t}\left\|\mathcal{T}\left(t-t_{k}\right)\right\|_{\mathcal{L}(E)}\left\|\left(\mathfrak{I}_{k} u_{n}\right)\left(t_{k}\right)-\left(\mathfrak{I}_{k} u\right)\left(t_{k}\right)\right\|\right] \\
\leq & M \int_{0}^{T}\left\|\left(\mathfrak{C} u_{n}\right)(s)-(\mathfrak{C} u)(s)\right\| d s+ \\
& M \sum_{0<t_{k}<T}\left\|\left(\mathfrak{I}_{k} u_{n}\right)\left(t_{k}\right)-\left(\mathfrak{I}_{k} u\right)\left(t_{k}\right)\right\| \\
\leq & M T^{1 / q}\left(\int_{0}^{T}\left\|\left(\mathfrak{C} u_{n}\right)(s)-(\mathfrak{C} u)(s)\right\|^{p} d s\right)^{1 / p} \\
& +M \sum_{0<t_{k}<T}\left\|\left(\mathfrak{I}_{k} u_{n}\right)\left(t_{k}\right)-\left(\mathfrak{I}_{k} u\right)\left(t_{k}\right)\right\|,
\end{aligned}
$$

and for $p=\infty$ we have

$$
\begin{aligned}
& \left\|\left(\mathfrak{F} u_{m}\right)(t)-(\mathfrak{F} u)(t)\right\| \\
\leq & \int_{0}^{t}\|\mathcal{T}(t-s)\|\left\|\left(\mathfrak{C} u_{n}\right)(s)-(\mathfrak{C} u)(s)\right\| d s \\
& +\sum_{0<t_{k}<T}\left\|\mathcal{T}\left(t-t_{k}\right)\right\|\left\|\left(\mathfrak{I}_{k} u_{n}\right)\left(t_{k}^{-}\right)-\left(\mathfrak{I}_{k} u\right)\left(t_{k}^{-}\right)\right\| \\
\leq \quad & M T \cdot \operatorname{ess} \sup \left\|\left(\mathfrak{C} u_{n}\right)(t)-(\mathfrak{C} u)(t)\right\| \\
& +M \sum_{0 \leq t \leq T}\left\|\left(\mathfrak{I}_{k} u_{n}\right)\left(t_{k}^{-}\right)-\left(\mathfrak{I}_{k} u\right)\left(t_{k}^{-}\right)\right\| .
\end{aligned}
$$

Using the continuity of the operators $\mathfrak{C}$ and $\mathfrak{I}_{k}$ it follows that for $1 \leq p \leq \infty$ we have that $\left\|\left(\mathfrak{F} u_{n}\right)(\cdot)-(\mathfrak{F} u)(\cdot)\right\|_{P C} \rightarrow 0$ as $n \rightarrow \infty$, so that $\mathfrak{F}: B \rightarrow B$ is a continuous operator. Since $B$ is a nonempty compact convex set, and $\mathfrak{F}: B \rightarrow B$ is a continuous operator, by Schauder's fixed point theorem it follows that there exists at least one $u(\cdot) \in B$ such that 


$$
u(t)=(\mathfrak{F} u)(t)=\mathcal{T}(t) u_{0}+\int_{0}^{t} \mathcal{T}(t-s)(\mathfrak{C} u)(s) d s+\sum_{0<t_{k}<t} \mathcal{T}\left(t-t_{k}\right)\left(\mathfrak{I}_{k} u\right)\left(t_{k}\right)
$$

for all $t \in[0, T]$; that is, $u(\cdot) \in B$ is a mild solution for (4).

Remark 2. It is easy to see that the conclusion Theorem 1 remains true if (6) is replaced by

$$
\|(\mathfrak{C} u)(t)\| \leq \xi\left(t,\|u(\cdot)\|_{P C}\right) \text {, for a.e. } t \in[0, T],
$$

and for each $u(\cdot) \in P C([0, T], E)$. The conclusion of Theorem 1 remains true if $(H 2)(b)$ is replaced by:

(H2) $\left(\boldsymbol{b}^{\prime}\right)$ For each bounded subset $B \subset P C([0, T], E)$ there exists $\gamma_{B}(\cdot) \in L^{1}\left([0, T], \mathbb{R}_{+}\right)$such that (7) holds and

$$
\chi(\mathcal{T}(t)(\mathfrak{C} B)(t)) \leq \int_{0}^{t} \gamma_{B}(s) \chi(B(s)) d s \text { for } t \in[0, T] .
$$

If $\{\mathcal{T}(t), t \geq 0\}$ is a compact $C_{0}$-semigroup or $\mathfrak{C}$ is a compact operator, then $\chi(\mathcal{T}(t)(\mathfrak{C} B)(t))=0$ for $t \in[0, T]$ and for each bounded set $B \subset P C([0, T], E)$ (see [48], Remark 8.2.1). Also, with a slight modification of the proof, the conclusion of Theorem 1 remains true if the condition $(H 2)(b)$ is replaced by:

(H2) $\left(\boldsymbol{b}^{\prime \prime}\right)$ For each bounded subset $B \subset P C([0, T], E)$ there exists $\gamma_{B}(\cdot) \in L^{1}\left([0, T], \mathbb{R}_{+}\right)$such that (7) holds and

$$
\chi((\mathfrak{C} B)(t)) \leq \gamma_{B}(t) \chi(B(t)) \text { for a.e. } t \in[0, T] .
$$

Next, suppose that $f(\cdot, \cdot):[0, T] \times E \rightarrow E$ is a function which satisfies the following condition:

(Hf) (a) $f:[0, T] \times E \rightarrow E$ is a Carathéodory function; that is, $t \mapsto f(t, u)$ is strongly measurable for all $u \in E, u \mapsto f(t, u)$ is continuous for a.e. $t \in[0, T]$, and there exist $a>0$ and $c(\cdot) \in L^{P}\left([0, T], \mathbb{R}_{+}\right)$such that $a M T<1-M \sum_{0<t_{k}<T} c_{k}$ and

$$
\|f(t, u)\| \leq c(t)+a\|u\|, t \in[0, T], u \in E,
$$

where $p \geq 1$.

(b) For each bounded set $B \subset E$ there exist $\gamma_{B}(\cdot) \in L^{1}\left([0, T], \mathbb{R}_{+}\right)$such that (7) holds and

$$
\chi(f(t, B)) \leq \gamma_{B}(t) \chi(B) \text { a.e. on }[0, T] .
$$

Then it is known (see [3], Theorem 3.1) that the operator $\mathfrak{C}$, defined by $(\mathfrak{C} u)(t):=f(t, u(t)), t \in[0, T]$, is a continuous operator from $L^{p}([0, T], E)$ into $L^{p}([0, T], E)$, and thus it is continuous from $P C([0, T], E)$ into $L^{p}([0, T], E)$. Moreover, $\mathfrak{C}$ is a causal operator and it satisfies $(H 2)(a)$ with $\xi(t, \eta):=c(t)+a \eta, t \in[0, T]$, $\eta \in \mathbb{R}_{+}$. Also, it is easy to see that $\mathfrak{C}$ satisfies $(H 2)\left(b^{\prime \prime}\right)$. We obtain the following result.

Corollary 1. Assume that $f:[0, T] \times E \rightarrow E$ satisfies (H1) and (Hf). If $A: D(A) \subset E \rightarrow E$ is the generator of a $C_{0}$-semigroup $\{\mathcal{T}(t) ; t \geq 0\}$, then the impulsive evolution equation

$$
\left\{\begin{array}{l}
u^{\prime}(t)=A u(t)+f(t, u(t)) \text { for } t \in[0, T] \backslash\left\{t_{1}, \ldots, t_{N}\right\} \\
u\left(t_{k}^{+}\right)=u\left(t_{k}^{-}\right)+\left(\Im_{k} u\right)\left(t_{k}^{-}\right) \\
u(0)=u_{0}
\end{array}\right.
$$

has at least one mild solution on $[0, T]$.

In the next result we show that, under the conditions (H1) and (H2), we can construct a sequence of successive approximations which converges to a mild solution of (4). 
Theorem 2. Assume that $\mathfrak{C}: P C([0, T], E) \rightarrow L^{p}([0, T], E)$ is a continuous causal operator such that the conditions (H1) and (H2) hold. If $A: D(A) \subset E \rightarrow E$ is the generator of a $C_{0}$-semigroup $\{\mathcal{T}(t) ; t \geq 0\}$, then there exists a sequence of functions $\left\{u_{n}(\cdot)\right\}_{n \geq 1}$ in $P C([0, T], E)$ such that $\left\|u_{n}(\cdot)-u(\cdot)\right\|_{P C} \rightarrow 0$ as $n \rightarrow \infty$, and $u(\cdot):[0, T] \rightarrow E$ is a mild solution for (4).

Proof. Let $r>0$ be such that (9) holds, and let $B_{0}$ and $\mathfrak{F}: B_{0} \rightarrow B_{0}$ be given by (10) and (11), respectively. We construct a sequence $\left\{u_{n}(\cdot)\right\}_{n \geq 1}$ of functions $u_{n}(\cdot) \in P C([0, T], E)$ as follows. Let $n \in \mathbb{N}$. For each $i \in\{1,2, \ldots, n\}$, we define

$$
u_{n}^{1}(t)=\mathcal{T}(t) u_{0}, \text { for } t \in[0, T / n]
$$

and

$$
u_{n}^{i}(t)=\left\{\begin{array}{l}
u_{n}^{i-1}(t), \text { for } t \in[0,(i-1) T / n] \\
\mathcal{T}(t) u_{0}+\int_{0}^{t-T / n} \mathcal{T}(t-s)\left(\mathfrak{C} u_{n}^{i-1}\right)(s) d s \\
+\sum_{0<t_{k}<t-\frac{T}{n}} \mathcal{T}\left(t-t_{k}\right)\left(\mathfrak{I}_{k} u_{n}^{i-1}\right)\left(t_{k}\right), \text { for } t \in((i-1) T / n, i T / n]
\end{array}\right.
$$

for $i>1$. Obviously, $\left\|u_{n}^{1}(t)\right\| \leq M\left\|u_{0}\right\| \leq r$ for $t \in[0, T / n]$. Let us suppose that $\left\|u_{n}^{i}(t)\right\| \leq r$ for $t \in[0, i T / n]$ and $i \in\{1,2, \ldots, v\}$ with $v \leq n-1$. Then we have

$$
\begin{aligned}
\left\|u_{n}^{\mathfrak{i}+1}(t)\right\| \leq & \left\|\mathcal{T}(t) u_{0}\right\|+M \int_{0}^{t-T / n}\left\|\left(\mathfrak{C} u_{n}^{i-1}\right)(s)\right\| d s \\
& +\sum_{0<t_{k}<t-\frac{T}{n}}\left\|\mathcal{T}\left(t-t_{k}\right)\left(\mathfrak{I}_{k} u_{n}^{i-1}\right)\left(t_{k}\right)\right\| \\
\leq & M\left\|u_{0}\right\|+\int_{0}^{t-T / n} \xi\left(s,\left\|u_{n}^{i-1}(s)\right\|\right) d s \\
& +M \sum_{0<t_{k}<t-\frac{T}{n}} c_{k}\left\|u_{n}^{i-1}\left(t_{k}\right)\right\| \\
\leq & M\left\|u_{0}\right\|+\int_{0}^{t-T / n} \xi(s, r) d s+M r \sum_{0<t_{k}<t-\frac{T}{n}} c_{k} \\
< & r
\end{aligned}
$$

for all $t \in[0,(i+1) T / n]$. Hence, by induction on $i$ we have that $\left\|u_{n}^{i}(t)\right\|<r$ for all $i \in\{1,2, \ldots, n\}$ and $t \in[0, i T / n]$. In the following, to simplify the notation, we put $u_{n}(\cdot)=u_{n}^{n}(\cdot), n \geq 1$. By the causality of $\mathfrak{C}$ and $\mathfrak{I}_{k}$, the sequence $\left\{u_{n}(\cdot)\right\}_{n \geq 1}$ can be written as

$$
u_{n}(t)=\left\{\begin{array}{l}
\mathcal{T}(t) u_{0} \text { if } t \in[0, T / n] \\
\mathcal{T}(t) u_{0}+\int_{0}^{t-T / n} \mathcal{T}(t-s)\left(\mathfrak{C} u_{n}\right)(s) d s \\
+\sum_{0<t_{k}<t-T / n} \mathcal{T}\left(t-t_{k}\right)\left(\mathfrak{I}_{k} u_{n}\right)\left(t_{k}^{-}\right) \text {if } t \in[T / n, T]
\end{array}\right.
$$

for every $n \geq 1$. Moreover, $u_{n}(\cdot) \in B_{0}$ for all $n \geq 1$. Next, if $0 \leq t \leq T / n$, then it is easy to see that

$$
\left\|\left(\mathfrak{F} u_{n}\right)(t)-u_{n}(t)\right\| \leq \int_{0}^{T / n}\left\|\mathcal{T}(t-s)\left(\mathfrak{C} u_{n}\right)(s)\right\| d s \leq M \int_{0}^{T / n} \psi(s) d s .
$$


If $T / n \leq t \leq T$, then we have

$$
\begin{aligned}
& \left\|\left(\mathfrak{F} u_{n}\right)(t)-u_{n}(t)\right\| \leq\left\|\int_{0}^{t} \mathcal{T}(t-s)\left(\mathfrak{C} u_{n}\right)(s) d s-\int_{0}^{t-T / n} \mathcal{T}(t-s)\left(\mathfrak{C} u_{n}\right)(s) d s\right\| \\
& +\left\|\sum_{0<t_{k}<t} \mathcal{T}\left(t-t_{k}\right)\left(\mathfrak{I}_{k} u_{n}\right)\left(t_{k}^{-}\right)-\sum_{0<t_{k}<t-T / n} \mathcal{T}\left(t-t_{k}\right)\left(\mathfrak{I}_{k} u_{n}\right)\left(t_{k}^{-}\right)\right\| \\
& \leq \int_{t-T / n}^{t}\|\mathcal{T}(t-s)\|_{\mathcal{L}(E)}\left\|\left(\mathfrak{C} u_{n}\right)(s)\right\| d s+\sum_{t-T / n<t_{k}<t}\left\|\mathcal{T}\left(t-t_{k}\right)\left(\mathfrak{I}_{k} u_{n}\right)\left(t_{k}^{-}\right)\right\| \\
& \leq M \int_{t-T / n}^{t}\left\|\left(\mathfrak{C} u_{n}\right)(s)\right\| d s+M \sum_{t-T / n<t_{k}<t}\left(\mathfrak{I}_{k} u_{n}\right)\left(t_{k}^{-}\right) \\
& \leq M \int_{t-T / n}^{t} \psi(s) d s+M \sum_{t-T / n<t_{k}<t}\left(\mathfrak{I}_{k} u_{n}\right)\left(t_{k}^{-}\right) .
\end{aligned}
$$

Therefore, we obtain that

$$
\left\|\left(\mathfrak{F} u_{n}\right)(\cdot)-u_{n}(\cdot)\right\|_{P C} \rightarrow 0 \text { as } n \rightarrow \infty .
$$

Let $V=\left\{u_{n}(\cdot) ; n \geq 1\right\}$. Since

$$
\left\|u_{n}(\cdot)\right\|_{P C} \leq\left\|u_{n}(\cdot)-\left(\mathfrak{F} u_{n}\right)(\cdot)\right\|_{P C}+\left\|\left(\mathfrak{F} u_{n}\right)(\cdot)\right\|_{P C},
$$

by (28) and the equicontinuity of $\mathfrak{F}(V)$ on $[0, T]$, it follows that $V$ is also equicontinuous on $[0, T]$. Define $V(t)=\left\{u_{n}(t) ; n \geq 1\right\}$ for $t \in[0, T]$. Then by the property of the measure of non-compactness we obtain

$$
\begin{aligned}
& \chi(V(t)) \leq \chi\left(\int_{0}^{t-T / n} \mathcal{T}(t-s)(\mathfrak{C} V)(s) d s+\sum_{0<t_{k}<t-T / n} \mathcal{T}\left(t-t_{k}\right)\left(\mathfrak{I}_{k} V\right)\left(t_{k}\right)\right) \\
& \leq \chi\left(\int_{0}^{t} \mathcal{T}(t-s)(\mathfrak{C} V)(s) d s\right)+\chi\left(\int_{t-T / n}^{t} \mathcal{T}(t-s)(\mathfrak{C} V)(s) d s\right) \\
& +\chi\left(\sum_{0<t_{k}<t-T / n} \mathcal{T}\left(t-t_{k}\right)\left(\mathfrak{I}_{k} V\right)\left(t_{k}\right)\right) .
\end{aligned}
$$

Let $t \in[0, T]$ be fixed and let $\varepsilon>0$. The we can find $n(\varepsilon) \geq 1$ such that $\int_{t-T / n}^{t} \psi(s) d s<\varepsilon / 2 M$ for $n \geq n(\varepsilon)$. Since

$$
\left\|\mathcal{T}(t-s)\left(\mathfrak{C} u_{n}\right)(s)\right\| \leq M\left\|\left(\mathfrak{C} u_{n}\right)(s)\right\| \leq M \psi(s)
$$

for a.e. $s \in[0, t]$ and $n \geq 1$, by Remark 1 we conclude that

$$
\begin{aligned}
& \chi\left(\int_{t-T / n}^{t} \mathcal{T}(t-s)(\mathfrak{C} V)(s) d s\right) \\
& =\chi\left(\left\{\int_{t-T / n}^{t} \mathcal{T}(t-s)\left(\mathfrak{C} u_{n}\right)(s) d s ; n \geq n(\varepsilon)\right\}\right) \\
& \leq 2 \sup _{n \geq n(\varepsilon)} M \int_{t-T / n}^{t} \psi(s) d s<\varepsilon .
\end{aligned}
$$

Using the last inequality and the fact that $\chi\left(\sum_{0<t_{k}<t-T / n} \mathcal{T}\left(t-t_{k}\right)\left(\Im_{k} V\right)\left(t_{k}\right)\right)=0$, we obtain that

$$
\chi(V(t)) \leq \chi\left(\int_{0}^{t} \mathcal{T}(t-s)(\mathfrak{C} V)(s) d s\right) .
$$


Since $V(t)$ is bounded, by Lemma 3 and (H2) it follows that

$$
\begin{aligned}
\chi(V(t)) & \leq \int_{0}^{t} \chi(\mathcal{T}(t-s)(\mathfrak{C} V)(s)) d s \\
& \leq \int_{0}^{t} \int_{0}^{s} M \gamma_{V}(\tau) \chi(V(\tau)) d \tau d s \\
& =\int_{0}^{t} \int_{\tau}^{t} M \gamma_{V}(\tau) \chi(V(\tau)) d s d \tau \\
& =\int_{0}^{t} M(t-\tau) \gamma_{V}(\tau) \chi(V(\tau)) d \tau \\
& \leq \int_{0}^{t} M T \gamma_{V}(\tau) \chi(V(\tau)) d \tau .
\end{aligned}
$$

Therefore, for each $t \in[0, T]$, we have

$$
v(t) \leq \int_{0}^{t} M T \gamma_{V}(t) v(t) d s
$$

where $v(t):=\chi(V(t), t \in[0, T]$. Then, by Gronwall's lemma, it follows that $v(t)=0$ for every $t \in[0, T]$, so that $\chi(V(t))=0$ for every $t \in[0, T]$. Moreover, since $\chi_{P C}(V)=\sup _{0 \leq t \leq T} \chi(V(t))$, hence $\chi_{P C}(V)=0$. Therefore, $V$ is a relatively compact subset of $B_{0}$. Then, by the Arzela-Ascoli theorem, and extracting a subsequence if necessary, we may assume that the sequence $\left\{u_{n}(\cdot)\right\}_{n \geq 1}$ converges uniformly on $[0, T]$ to a continuous function $u(\cdot) \in B_{0}$. Since

$$
\begin{aligned}
\|(\mathfrak{F} u)(\cdot)-u(\cdot)\|_{P C} \leq & \left\|(\mathfrak{F} u)(\cdot)-\left(\mathfrak{F} u_{n}\right)(\cdot)\right\|_{P C}+\left\|\left(\mathfrak{F} u_{n}\right)(\cdot)-u_{n}(\cdot)\right\|_{P C} \\
& +\left\|u_{n}(\cdot)-u(\cdot)\right\|_{P C}
\end{aligned}
$$

by the continuity of $\mathfrak{F}$ and (28), we get $\|(\mathfrak{F} u)(\cdot)-u(\cdot)\|_{P C}=0$. It follows that

$$
u(t)=(\mathfrak{F} u)(t)=\mathcal{T}(t) u_{0}+\int_{0}^{t} \mathcal{T}(t-s)(\mathfrak{C} u)(s) d s+\sum_{0<t_{k}<t} \mathcal{T}\left(t-t_{k}\right)\left(\mathfrak{I}_{k} u\right)\left(t_{k}\right)
$$

for all $t \in[0, T]$; that is, $u(\cdot)$ is a mild solution of the causal evolution Equation (4).

\section{Applications}

1. Consider the following impulsive integro-differential evolution equation

$$
\left\{\begin{array}{l}
u^{\prime}(t)=A u(t)+\int_{0}^{t} K(t, s) f(s, u(s)) d s \text { for a.e. } t \in[0, T] \\
u\left(t_{k}^{+}\right)=u\left(t_{k}^{-}\right)+\left(\Im_{k} u\right)\left(t_{k}^{-}\right), k=1,2, \ldots, N \\
u(0)=u_{0}
\end{array}\right.
$$

where $f:[0, T] \times E \rightarrow E$ satisfies condition (Hf) and $K:[0, T] \times[0, T] \rightarrow \mathcal{L}(E)$ is strongly continuous. Put

$$
(\mathfrak{C} u)(t):=\int_{0}^{t} K(t, s) f(s, u(s)) d s, t \in[0, T] .
$$

It is well known that $\mathfrak{C}$ defines a continuous operator from $L^{p}([0, T], E)$ into itself (see ([49], Proposition 9.5.2) or ([50], p. 160)). Then for each $u(\cdot) \in P C([0, T], E)$ we have 


$$
\begin{aligned}
\|(\mathfrak{C} u)(t)\| & \leq \int_{0}^{t}\|K(s, \tau)\|_{\mathcal{L}(E)}\|f(\tau, u(\tau))\| d \tau \\
& \leq M_{1} \int_{0}^{t}[c(\tau)+a\|u(\tau)\|] d \tau \\
& \leq \xi\left(t,\|u(\cdot)\|_{P C}\right):=M_{1} \int_{0}^{t} c(\tau) d \tau+a M_{1} T\|u(\cdot)\|_{P C},
\end{aligned}
$$

where $M_{1}:=\sup \left\{\|K(t, s)\|_{\mathcal{L}(E)} ; t, s \in[0, T]\right\}$. Next, if $B$ is a bounded set in $P C([0, T], E)$, then it is easy to show that $\mathfrak{C} B$ is bounded and equicontinuous on $[0, T]$ (as a subset of $P C([0, T], E)$ ). Thus, by (25) and Theorem 1.2.2 in [51], we have

$$
\begin{aligned}
\chi((\mathfrak{C} B)(t)) & =\chi\left(\int_{0}^{t} K(t, s) f(s, B(s)) d s\right) \leq \int_{0}^{t} \chi(K(t, s) f(s, B(s))) d s \\
& \leq \int_{0}^{t} M_{1} \gamma_{B}(s) \chi(B(s)) d s, t \in[0, T] .
\end{aligned}
$$

Consequently, the operator $\mathfrak{C}$, defined by (30), is a continuous causal operator from $P C([0, T], E)$ into $L^{p}([0, T], E)$ and it satisfies (24) and (8). Assume that hypotheses (H1), (Hf) hold, $\int_{0}^{T} M_{1} \gamma_{B}(t) d t<$ $\frac{1}{2 M T}$ and $K:[0, T] \times[0, T] \rightarrow \mathcal{L}(E)$ is strongly continuous. Then, by Theorem 1 , it follows that (29) has at least one mild solution in $P C([0, T], E)$ provided that (5) holds.

2. Consider the following impulsive reaction diffusion equation

$$
\left\{\begin{array}{l}
\frac{\partial z}{\partial t}(t, x)=\frac{\partial^{2} z}{\partial x^{2}}(t, x)+\int_{0}^{t} k(t, s) f(s, z(s, x)) d s, x \in(0, \pi), t \in[0, T] \backslash\left\{t_{1}, . ., t_{N}\right\} \\
z\left(t_{k}^{+}, x\right)-z\left(t_{k}^{-}, x\right)=\int_{0}^{x} z\left(t_{k}^{-}, y\right) g_{k}(x) d y, \quad x \in(0, \pi), k=1, \ldots, N \\
z(t, 0)=z(t, \pi)=0, \quad t \in[0, T] \\
z(0, x)=z_{0}(x), \quad x \in(0, \pi)
\end{array}\right.
$$

where $0=t_{0}<t_{1}<t_{2}<\ldots<t_{N}<t_{N+1}=T, z\left(t_{k}^{+}, x\right)=\lim _{(h, x) \rightarrow\left(0^{-}, x\right)} z\left(t_{k}+h, x\right), z\left(t_{k}^{-}, x\right)=$ $\lim _{(h, x) \rightarrow\left(0^{-}, x\right)} z\left(t_{k}+h, x\right), f(\cdot, \cdot):[0, T] \times[0, \pi] \rightarrow \mathbb{R}$ and $k(\cdot, \cdot):[0, T] \times[0, T] \rightarrow \mathbb{R}$ are given functions, $z_{0}(\cdot), g_{k}(\cdot) \in E:=L^{2}[0, \pi]$ and $k=1, \ldots, N$. Also, we assume that $\left\|\pi_{h} g_{k}-g_{k}\right\|_{L^{2}[0, \pi-h]} \rightarrow 0$ as $h \rightarrow 0^{+}$ for $k=1, \ldots, N$, where $\left(\pi_{h} g_{k}\right)(t)=g_{k}(t+h)$. We can show that problem (31) is an abstract formulation of problem (29). For this, let

$$
\begin{aligned}
u(t)(x): & =z(t, x),(t, x) \in[0, T] \times[0, \pi], \\
\left(\left(\mathfrak{I}_{k} u\right)(t)\right)(x): & =\int_{0}^{x} u(t)(y) g_{k}(x) d y,(t, x) \in[0, T] \times[0, \pi], k=1,2, \ldots, N, \\
(\mathfrak{C} u)(t): & =\int_{0}^{t} K(t, s) F(s, u(s)) d s, t \in[0, T],
\end{aligned}
$$

where $F(\cdot, \cdot):[0, T] \times E \rightarrow E$ is given by $F(t, u(\cdot))(x):=f(t, z(\cdot, x))$ for $(t, x) \in[0, T] \times[0, \pi]$, and $K(\cdot, \cdot):[0, T] \times[0, T] \rightarrow \mathcal{L}(E)$ is given by $(K(t, s) u(\cdot))(x):=k(t, s) u(\cdot)(x)=k(t, s) z(\cdot, x)$ for $t, s \in[0, T], x \in[0, \pi]$. Next, let $t \geq 0$ and let us define $\mathcal{T}(t): E \rightarrow E$ by

$$
(\mathcal{T}(t) u(\cdot))(x):=\sum_{n=0}^{\infty} a_{n}(u(\cdot)) e^{-n^{2} t} \sin n x
$$

for each $u(\cdot) \in E$, where

$$
a_{n}(u(\cdot))=\sqrt{\frac{2}{\pi}} \int_{0}^{\pi} u(\cdot)(x) \sin n x d x .
$$


Then it is known (see ([41], Problem 4.2 and Problem 7.8)) that $\{\mathcal{T}(t), t \geq 0\}$ is a compact $C_{0}$-semigroup and its infinitesimal generator $A: D(A) \subset E \rightarrow E$ is given by

$$
(A u(\cdot))(x)=-\sum_{n=1}^{\infty} n^{2} a_{n}(u(\cdot)) \sin n x, u(\cdot) \in D(A),
$$

where $D(A)$ is the space of all functions $u(\cdot) \in E$ such that $u(\cdot), u^{\prime}(\cdot)$ are absolutely continuous, $u^{\prime \prime}(\cdot) \in E$ and $u(\cdot)(0)=u(\cdot)(\pi)=0$. Also, there exists $M>1$ such that $\|\mathcal{T}(t)\|_{\mathcal{L}(E)} \leq M$ for $t \in[0, T]$. From the above it follows that (31) can be written in the abstract form (29). Now, assume that

(f) $f(\cdot, \cdot):[0, T] \times[0, \pi] \rightarrow \mathbb{R}$ is a Carathéodory function; that is, $t \mapsto f(t, x)$ is measurable for all $x \in[0, \pi], x \mapsto f(t, x)$ is continuous for a.e. $t \in[0, T]$, and there exist $a>0$ and $c(\cdot) \in L^{2}\left([0, T], \mathbb{R}_{+}\right)$

$$
\|f(t, x)\| \leq c(t)+a\|x\|, t \in[0, T], x \in[0, \pi] .
$$

(k) $k(\cdot, \cdot):[0, T] \times[0, T] \rightarrow \mathbb{R}$ is continuous.

Then it is easy to check that $F(\cdot, \cdot)$ verifies the condition (Hf) and $K(\cdot, \cdot)$ is strongly continuous. Since $\{\mathcal{T}(t), t \geq 0\}$ is a compact $C_{0}$-semigroup, for any bounded set $B \subset P C([0, T], E)$, we have $\chi(\mathcal{T}(t)(\mathfrak{C} B)(t))=0$ for $t \in[0, T]$. It remains to show that $\mathfrak{I}_{k}$ is a compact operator for each $k=1, \ldots, N$. For this, we must show that for any bounded set $B \subset P C([0, T], E), \Im_{k} B$ is equicontinuos and $\left(\Im_{k} B\right)(t) \subset$ $L^{2}[0, T]$ is relatively compact for every $t \in[0, T]$. For any $u(\cdot), v(\cdot) \in B$, using Hölder's inequality, we have

$$
\begin{aligned}
\|\left(\mathfrak{I}_{k} u\right)(t)-\left(\mathfrak{I}_{k} v\right) & (t)\left\|_{L^{2}[0, T]}=\right\| \int_{0}^{x} u(t)(y) g_{k}(x) d y-\int_{0}^{x} v(t)(y) g_{k}(x) d y \|_{L^{2}[0, T]} \\
& \leq \int_{0}^{x}\left\|[u(t)(y)-v(t)(y)] g_{k}(x)\right\|_{L^{2}[0, T]} d y \\
& \leq \int_{0}^{\pi}\left(\int_{0}^{\pi}|u(t)(y)-v(t)(y)|^{2}\left|g_{k}(x)\right|^{2} d x\right)^{1 / 2} d y \\
& =\left(\int_{0}^{\pi}|u(t)(y)-v(t)(y)| d y\right)\left\|g_{k}\right\|_{L^{2}[0, T]} \\
& \leq\left(\int_{0}^{\pi} d y\right)^{1 / 2}\left(\int_{0}^{\pi}|u(t)(y)-v(t)(y)|^{2} d y\right)^{1 / 2}\left\|g_{k}\right\|_{L^{2}[0, T]} \\
& =\sqrt{\pi}\left\|g_{k}\right\|_{L^{2}[0, T]}\|u(t)-v(t)\|_{L^{2}[0, T]}
\end{aligned}
$$

that is,

$$
\left\|\left(\mathfrak{I}_{k} u\right)(t)-\left(\mathfrak{I}_{k} v\right)(t)\right\|_{L^{2}[0, T]} \leq c_{k}\|u(t)-v(t)\|_{L^{2}[0, T]},
$$

for every $u(\cdot), v(\cdot) \in B$, where $c_{k}=\sqrt{\pi}\left\|g_{k}\right\|_{L^{2}[0, T]}$. From the above inequality it follows that

$$
\left\|\mathfrak{I}_{k} u-\mathfrak{I}_{k} v\right\|_{P C} \leq c_{k}\|u-v\|_{P C}
$$

for every $u(\cdot), v(\cdot) \in B$, and so, $\Im_{k} B$ is equicontinuous. Using the compactness result from ([52], p. 74), $\left(\mathfrak{I}_{k} B\right)(t) \subset L^{2}[0, T]$ is relatively compact if and only if $\left(\mathfrak{I}_{k} B\right)(t)$ is bounded and

$$
\left\|\pi_{h}\left(\mathfrak{I}_{k} u\right)(t)-\left(\mathfrak{I}_{k} u\right)(t)\right\|_{L^{2}[0, \pi-h]} \rightarrow 0 \text { as } h \rightarrow 0^{+}
$$

for every $u(\cdot) \in B$. The boundedness of $\left(\mathfrak{I}_{k} B\right)(t)$ follows from (32) and the definition of the causal operator. More exactly, we have that

$$
\left\|\left(\mathfrak{I}_{k} u\right)(t)\right\|_{L^{2}[0, T]} \leq c_{k}\|u(t)\|_{L^{2}[0, T]} \leq c_{k}\|u\|_{P C}<c r
$$

for every $u(\cdot) \in B$, where $r:=\sup _{u(\cdot) \in B}\|u\|_{P C}$ and $c:=\sum_{k=1}^{N} c_{k}$. 
Using the Hölder inequality it is not difficult to show that

$$
\begin{aligned}
& \left\|\pi_{h}\left(\mathfrak{I}_{k} u\right)(t)-\left(\mathfrak{I}_{k} u\right)(t)\right\|_{L^{2}[0, \pi-h]}=\left(\int_{0}^{\pi-h}\left|\left(\mathfrak{I}_{k} u\right)(t)(x+h)-\left(\mathfrak{I}_{k} u\right)(t)(x)\right|^{2} d x\right)^{1 / 2} \\
& =\left[\int_{0}^{\pi-h}\left(\int_{0}^{x+h} u(t)(y) g_{k}(x+h) d y-\int_{0}^{x} u(t)(y) g_{k}(x) d y\right)^{2} d x\right]^{1 / 2} \\
& =\left[\int_{0}^{\pi-h}\left(\int_{0}^{x} u(t)(y)\left[g_{k}(x+h)-g_{k}(x)\right] d y+\int_{x}^{x+h} u(t)(y) g_{k}(x+h) d y\right)^{2} d x\right]^{1 / 2} \\
& \leq 2 \sqrt{\pi}\|u\|_{P C}\left\|\pi_{h} g_{k}-g_{k}\right\|_{L^{2}[0, \pi-h]}+2\left(\int_{x}^{x+h} u(t)(y) d y\right)\left\|g_{k}(\cdot+h)\right\|_{L^{2}[0, \pi-h]} .
\end{aligned}
$$

Using the last estimation and the fact that $\left\|\pi_{h} g_{k}-g_{k}\right\|_{L^{2}[0, \pi-h]} \rightarrow 0$ as $h \rightarrow 0^{+}$, we obtain (33). Since, for each $k=1, \ldots, N$, we showed that for any bounded set $B \subset P C([0, T], E), \mathfrak{I}_{k} B$ is equicontinuous and $\left(\Im_{k} B\right)(t) \subset L^{2}[0, T]$ is relatively compact for every $t \in[0, T]$, it follows that $\Im_{k}$ is a compact operator for each $k=1, \ldots, N$. Consequently, if $2 M c<1$, then all the conditions of Theorem 1 are satisfied, so that $(31)$ has a solution $z(\cdot, \cdot)$ on $[0, T] \times[0, \pi]$.

\section{Conclusions}

The theory of impulsive evolution differential equations with causal operators is an important one because it covers a large class of different types of impulsive evolution differential equations. The study of these evolution equations hopefully will be continued with impulsive evolution equations with nonlinear operators or impulsive evolution differential inclusions involving causal operators. Another direction of investigation is to study fractional differential equations with causal operators and their applications.

Author Contributions: All authors contributed equally. All authors have read and agreed to the published version of the manuscript.

Funding: This research received no external funding.

Conflicts of Interest: The authors declare no conflict of interest.

\section{References}

1. Tonelli, L. Sulle equazioni funzionali di Volterra. Bull. Calcutta Math. Soc. 1930, 20, 31-48.

2. Tychonoff, A. Sur les equations fonctionnelles de Volterra et leurs applications a certains problemes de la Physique Mathematique. Mosc. Univ. Math. Bull. 1938, A1,1-25.

3. Appell, J.; Zabreǐko, P.P. Nonlinear Superposition Operators; Cambridge University Press: Cambridge, UK, 1990.

4. Corduneanu, C. Functional Equations with Causal Operators; Taylor and Francis: London, UK; New York, NY, USA, 2002.

5. Azbelev, N.V.; Maksimov, V.P.; Rakhmatullina, L.F. Introduction to The Theory of Linear Functional Differential Equations; World Federation Publishers Company, Inc.: Atlanta, GA, USA, 1995.

6. Gil', M.I. Stability of Neutral Functional Differential Equations; Atlantis Press: Amsterdam, The Netherlands, 2014.

7. Gripenberg, G.; Londen, S.O.; Staffans, O. Volterra Integral and Functional Equations; Cambridge University Press: Cambridge, UK, 1990.

8. Kurbatov, V.G. Functional Differential Operators and Equations; Kluwer Academic Publishers: Dordrecht, The Netherlands, 1999.

9. O'Regan, D.; Precup, R. Existence criteria for integral equations in Banach spaces. J. Inequalities Appl. 2001, 6, 77-97. [CrossRef]

10. Li, Y.; Mahdavi, M. Linear and Quasilinear Equations with Abstract Volterra Operators; Volterra Equations and Applications; Gordon\&Breach: Reading, UK, 2000.

11. Lakshmikantham, V.; Leela, S.; Drici, Z.; McRae, F.A. Theory of Causal Differential Equations; Atlantis Studies in Mathematics for Engineering and Science; World Scientific: Singapore, 2010; Volume 5.

12. Mahdavi, M. Contribution to the Theory of Functional Differential Equations Involving Abstract Volterra Operators. Ph.D. Thesis, University of Texas, Arlington, TX, USA, 1992. 
13. Ahangar, R. Nonanticipating dynamical model and optimal control. Appl. Math. Lett. 1989, 2, 15-18. [CrossRef]

14. Corduneanu, C. A modified LQ-optimal control problem for causal functional differential equations. Nonlinear Dyn. Syst. Theory 2004, 4, 139-144.

15. Corduneanu, C. Boundedness of solutions for a second order differential equation with causal operators. Nonlinear Stud. 2011, 18, 135-139.

16. Corduneanu, C.; Mahdavi, M. Neutral functional equations with causal operators on a semi-axis. Nonlinear Dyn. Syst. Theory 2008, 8, 339-348.

17. Ilchmann, A.; Ryan, E.P.; Sangwin, C.J. Systems of controlled functional differential equations and adaptive tracking. Siam J. Control Optim. 2002, 40, 1746-1764. [CrossRef]

18. Mahdavi, M. Asymptotic Behavior in Some Classes of Functional Differential Equations. Nonlinear Dyn. Syst. Theory 2004, 4, 51-57.

19. Martynyuk, A.A.; Martynyuk-Chernienko, Y.A. Analysis of the set of trajectories of nonlinear dynamics: Equations with causal robust operator. Differ. Equ. 2015, 51, 11-22. [CrossRef]

20. Ryan, E.P.; Sangwin, C.J. Controlled functional differential equations and adaptive tracking. Syst. Control. Lett. 2002, 47, 365-374. [CrossRef]

21. Kwapisz, M. Remarks on the existence and uniqueness of solutions of Volterra functional equations in Lp-space. J. Integr. Appl. 1991, 3, 383-392. [CrossRef]

22. Kwapisz, M. Bielecki's method; Existence and uniqueness results for Volterra integral equations in Lp-space. J. Math. Anal. Appl. 1991, 154, 403-416. [CrossRef]

23. McNabb, A.; Weir, G. Comparison theorems for causal functional differential equations. Proc. Am. Math. Soc. 1988, 104, 449-452.

24. Meehan, M.; O'Regan, D. Existence theory for nonlinear Volterra integrodifferential and integral equations. Nonlinear Anal. 1998, 31, 317-341. [CrossRef]

25. O'Regan, D. Abstract volterra equations. Panam. Math. J. 1997, 7, 19-28.

26. Drici, Z.; McRae, F.A.; Devi, J.V. Monotone iterative technique for periodic boundary value problems with causal operators. Nonlinear Anal. 2006, 64, 1271-1277. [CrossRef]

27. Drici, Z.; McRae, F.A.; Devi, J.V. Differential equations with causal operators in a Banach space. Nonlinear Anal. 2005, 62, 301-313. [CrossRef]

28. Gil', M.I. Positive solutions of equations with nonlinear causal mappings. Positivity 2007, 11, 523-535. [CrossRef]

29. Lupulescu, V.; Causal functional differential equations in Banach spaces. Nonlinear Anal. 2008, 69, 4787-4795. [CrossRef]

30. Lupulescu, V. On a class of functional differential equations in Banach spaces. Electron. J. Qual. Theory Differ. 2010, 64, 1-17. [CrossRef]

31. Obukhovskii, V.; Zecca, P. On certain classes of functional inclusions with causal operators in Banach spaces. Nonlinear Anal. 2011, 74, 2765-2777. [CrossRef]

32. Gil', M.I. $L^{2}$-stability of vector equations with nonlinear causal operator. Dyn. Syst. Appl. 2008, 17, $201-220$.

33. Jankowski, T. Boundary value problems with causal operators. Nonlinear Anal. 2008, 68, 3625-3632. [CrossRef]

34. Jankowski, T. Nonlinear boundary value problems for second order differential equations with causal operators. J. Math. Appl. 2007, 332, 1380-1392. [CrossRef]

35. Geng, F. Differential equations involving causal operators with nonlinear periodic boundary conditions. Math. Comput. 2008, 48, 859-866. [CrossRef]

36. Yakar, A.; Koksal, M.E. Quasilinearization method for nonlinear differential equations with causal operators. J. Nonlinear Sci. Appl. 2016, 9, 1356-1364. [CrossRef]

37. Wang, W.L.; Tian, J.F. Generalized monotone iterative method for nonlinear boundary value problems with causal operators. Bound. Value Probl. 2014, 2014, 192. [CrossRef]

38. Wang, W.L.; Tian, J.F. Difference equations involving causal operators with nonlinear boundary conditions. J. Nonlinear Sci. Appl. 2015, 8, 267-274. [CrossRef]

39. Zhao, Y.; Song, G.; Sun, X. Integral boundary value problems with causal operators. Comput. Math. Appl. 2010, 59, 2768-2775. [CrossRef] 
40. Agarwal, R.P.; Arshad, S.; Lupulescu, V.; O’Regan, D. Evolution equations with causal operators. Differ. Equ. Appl. 2015, 7, 15-26. [CrossRef]

41. Vrabie, I.I. Co-Semigroups and Applications; North-Holland Publishing Co.: Amsterdam, The Netherlands, 2003.

42. Pazy, A. Semigroups of Linear Operators and Applications for Partial Differential Equations; Springer: New York, NY, USA, 1983.

43. Kamenskii, M.; Obukhovskii, V.; Zecca, P. Condensing Multivalued Maps and Semilinear Differential Inclusions in Banach Spaces; de Gruyter Series in Nonlinear Analysis and Applications; Walter de Gruyter: Berlin, Germany; New York, NY, USA, 2001; Volume 7.

44. Kuratowski, K. Sur les espaces complets. Fundamenta Mathematicae 1930, 15, 301-309. [CrossRef]

45. Hernández, E.; Pierri, M.; Gonçalves, G. Existence results for an impulsive abstract partial differential equation with state-dependent delay. Comput. Math. Appl. 2006, 52, 411-420. [CrossRef]

46. Bothe, D. Multivalued perturbation of m-accretive differential inclusions. Israel J. Math. 1998, 108, $109-138$. [CrossRef]

47. Kisielewicz, M. Multivalued differential equations in separable Banach spaces. J. Optim. Theory Appl. 1982, 37, 231-249. [CrossRef]

48. Cârjă, O.; Necula, M.; Vrabie, I.I. Viability, Invariance and Applications; North-Holland Mathematics Studies,; Elsevier Science B.V.: Amsterdam, The Netherlands, 2007; Volume 207.

49. Edwards, R.E. Functional Analysis-Theory and Applications, Holt; Rinehart and Winston, Inc.: New York, NY, USA, 1965.

50. Szufla, S. On the existence of Lp-solutions of Volterra integral equations in Banach spaces. Funkcialaj Ekvacioj 1984, 27, 157-172.

51. Guo, D.; Lakshmikantham, V.; Liu, X. Nonlinear Integral Equations in Abstract Spaces; Kluwer Academic Publishers: Dordrecht, The Netherlands, 1996.

52. Simon, J. Compact sets in the space $L^{p}(0, T ; B)$. Ann. Math. Pura Appl. 1987, 154, 65-96.

(C) 2019 by the authors. Licensee MDPI, Basel, Switzerland. This article is an open access article distributed under the terms and conditions of the Creative Commons Attribution (CC BY) license (http:/ / creativecommons.org/licenses/by/4.0/). 